\title{
GAME THEORY AND COST ALLOCATION PROBLEMS*
}

\author{
S. H. TIJS AND T. S. H. DRIESSEN \\ Department of Mathematics, University of Nijmegen, Toernooiveld, \\ 6525 ED Nijmegen, The Netherlands \\ Department of Applied Mathematics, Twente University of Technology, \\ 7500 AE Enschede, The Netherlands
}

\begin{abstract}
Problems of allocating joint costs in a reasonable way arise in many practical situations where people decide to work together to save costs. Cost allocation methods based on game theoretical concepts take into account the strategic aspects of cost allocation situations. We give a survey of cost allocation methods based on the nucleolus and the Shapley value, and introduce also a new one, the so-called cost gap allocation method which is based on the $\tau$-value. It is shown that for some large subclasses of cost allocation problems this new cost allocation method coincides with old separable cost methods proposed in the thirties by the Tennessee Valley Authority and also with the separable costs-remaining benefits (SCRB) method. Properties of this cost gap allocation method are also treated.
\end{abstract}

(COST ALLOCATION METHOD)

\section{Introduction}

Cost allocation problems arise in many real life situations, where individuals, all with their own purposes, decide to work together. In these situations the problem arises how to divide among the participants the joint costs (and implicitly the cost savings) which result from the cooperation. There is a whole literature on this subject with all kinds of proposals to deal with the cost allocation problem. Many of the proposals ignore the strategic aspects of such situations. A natural framework to study cost allocation problems is game theory and it is not surprising that there are now many papers dealing with cost allocation methods based on game theoretical concepts. An extensive list of such papers is given in the references at the end of this paper. In this study we shall concentrate on game theoretical cost allocation methods.

One has to realize beforehand that in theoretical studies one cannot expect a final answer to the question: What is the best cost allocation method? The choice of a method depends on the concrete situation where such a cost allocation problem arises, the ideas of the participants with respect to fairness, the power feelings of the participants, the difficulty of understanding and calculating a cost allocation proposal, and many other factors.

It is a task of game theorists to design for classes of cost allocation problems a number of cost allocation methods and to describe the mathematical properties of such methods. The properties can reflect ethical, economic or political aspects. The comparison of cost allocation methods can then be based on properties, which the methods possess or fail to possess. Also secondary considerations, such as easiness to understand or to calculate, play a role in practice.

The cost allocation problem is comparable with the problem in social choice theory to find a suitable welfare function or social choice rule. It is also comparable with the problem of choosing a suitable statistical test in statistics. In specific cost allocation situations, persons involved or arbitrators have to decide which method is most suitable for their purposes. They have to put priorities on the list of desirable properties for the cost allocation method. If one wants a method with too many properties, then impossibilities arise just as in social choice theory (see Arrow 1963).

\footnotetext{
* Accepted by Ambar G. Rao; received May 1, 1985.
} 
The main purpose of this paper is to introduce a new cost allocation method, the so-called cost gap method, for a class of cost allocation problems and to study the properties of this new method. It will turn out that some separable cost allocation methods, developed during the 1930's by the engineers and economists of the Tennessee Valley Authority (see Heaney 1979, Ransmeier 1942, Straffin and Heaney 1981) can be seen as rough forms of this cost gap method.

The paper is organized as follows. In $\S 2$ we list several desirable properties for cost allocation methods and we give a survey of three well-known types of cost allocation methods. Two of the three treated types of methods are based on well-known game theoretical concepts, while the third method is closely related to the new cost gap method, which is considered in \$3. In the last section, some examples are given to illustrate the methods treated in $\$ \S 2$ and 3. The examples include cost allocation problems arising at airports or in networks.

\section{Cost Allocation Methods and Their Properties}

To work in a game theoretical context, a cost allocation problem is transformed into a cost game. This is a pair $\langle N, c\rangle$ where $N:=\{1,2, \ldots, n\}$ denotes the set of participants, each of them indexed by a number. The elements of $N$ are called players and subsets of $N$ are called coalitions. Further, the cost function $c: 2^{N} \rightarrow \mathbb{R}$ assigns to any nonempty coalition $S$ the minimal costs $c(S)$ which should be involved if the individuals in $S$ should work together in order to serve their own purposes. For the empty coalition one puts $c(\varnothing)=0$. This interpretation of the cost function $c$ implies that $c$ is subadditive, i.e. $c(S \cup T) \leq c(S)+c(T)$ for all $S, T \subset N$ with $S \cap T=\varnothing$. The set of all subadditive cost functions corresponding to $n$-person cost games will be denoted by $C G_{n}$. Let $A_{n}$ be a subset of $C G_{n}$.

A cost allocation method on $A_{n}$ is a map $M: A_{n} \rightarrow \mathbb{R}^{n}$. For each cost function $c \in A_{n}$, the $i$ th coordinate $M_{i}(c)$ of the cost vector $M(c)=\left(M_{1}(c), M_{2}(c), \ldots, M_{n}(c)\right)$ represents the costs that the method $M$ charges to player $i$. For a cost allocation method all kinds of properties which can be desirable for the participants are listed below. There is, however, no method $M: A_{n} \rightarrow \mathbb{R}^{n}$ which possesses all these properties.

(i) $M: A_{n} \rightarrow \mathbb{R}^{n}$ is said to be an efficient cost allocation method if for each $c \in A_{n}$ we have $\sum_{i \in N} M_{i}(c)=c(N)$.

(ii) $M: A_{n} \rightarrow \mathbb{R}^{n}$ is said to be an individually rational cost allocation method if for each $c \in A_{n}$ we have $M_{i}(c) \leq c(\{i\})$ for all $i \in N$.

(iii) $M: A_{n} \rightarrow \mathbb{R}^{n}$ is said to be a stable cost allocation method if for each $c \in A_{n}$ with $\operatorname{CORE}(c) \neq \varnothing$ we have $M(c) \in \operatorname{CORE}(c)$, where the core of $c$ is given by

$$
\operatorname{CORE}(c):=\left\{y \in \mathbb{R}^{n} ; \sum_{i \in N} y_{i}=c(N) \text { and } \sum_{i \in S} y_{i} \leq c(S) \text { for all } S \neq \varnothing\right\} \text {. }
$$

(iv) $M: A_{n} \rightarrow \mathbb{R}^{n}$ is said to possess the dummy player property if $M_{i}(c)=c(\{i\})$ for all $c \in A_{n}$ and all $i \in N$ for which $c(S \cup\{i\})-c(S)=c(\{i\})$ for all $S \subset N-\{i\}$.

(v) $M: A_{n} \rightarrow \mathbb{R}^{n}$ is said to possess the anonymity property if for each $c \in A_{n}$ and each permutation $\theta: N \rightarrow N$ with $\theta c \in A_{n}$ we have $M_{\theta(i)}(\theta c)=M_{i}(c)$ for all $i \in N$, where $\theta c$ is the cost function given by $(\theta c)(\theta S)=c(S)$ for all $S \subset N$.

(vi) $M: A_{n} \rightarrow \mathbb{R}^{n}$ is said to be an aggregate monotonic cost allocation method if for each $c^{1}, c^{2} \in A_{n}$ with $c^{2}(S)=c^{1}(S)$ for all $S \neq N$ and $c^{2}(N) \geq c^{1}(N)$, we have $M_{i}\left(c^{2}\right)$ $\geq M_{i}\left(c^{1}\right)$ for all $i \in N$.

In the remainder of the paper several other properties will also be considered. Let us make some remarks and describe then some well-known cost allocation methods. First we note that for many cost functions $c \in C G_{n}$ its core is empty. Further we note that for any cost allocation method on the set of cost functions with a nonempty core, stability implies efficiency as well as individual rationality. 
The simplest cost allocation method on $C G_{n}$ is the egalitarian method $E$ which apportions the joint $\operatorname{cost} c(N)$ of the grand coalition equally to the players, i.e. $E_{i}(c)$ $=c(N) / n$ for each $c \in C G_{n}$ and each $i \in N$. This egalitarian method is efficient, monotonic in the aggregate and possesses also the anonymity property, but it fails to take strategic aspects into consideration: $E$ is not individually rational and does not possess the dummy player property.

Now we describe three cost allocation methods, which have obtained much attention in the literature and which are applied to several practical situations.

\section{(1) Methods Based on Marginal Costs in Entering Coalitions}

The key idea of these methods was introduced by Shapley (1953). Let $c \in C G_{n}$ be a cost function and $\theta: N \rightarrow N$ a permutation of the grand coalition. With respect to $\theta$, the formation of the grand coalition $N$ can be seen as the sequential process, where the players form the grand coalition by entering one by one in the order $\theta(1), \theta(2)$, ..., $\theta(n)$. Let any player pay the additional costs which arise by joining his predecessors, i.e. player $\theta(i)$ has to pay the marginal cost $M_{\theta(i)}^{\theta}(c)$ given by

$$
M_{\theta(i)}^{\theta}(c)=c(\{\theta(1), \theta(2), \ldots, \theta(i)\})-c(\{\theta(1), \theta(2), \ldots, \theta(i-1)\}) .
$$

Then the corresponding cost allocation method $M^{\theta}$ on $C G_{n}$ is efficient. It possesses the dummy player property, but it is not anonymous. The Shapley cost allocation method $\Phi$ is obtained by averaging in a uniform manner these cost allocation methods $M^{\theta}$ corresponding to the various permutations $\theta$ on $N$. Thus $\Phi=(n !)^{-1} \sum_{\theta} M^{\theta}$ where the sum is taken over all permutations on $N$. Due to this sum, the Shapley method is anonymous, but it fails to be stable. The Shapley method is based on the Shapley value, a game theoretical concept introduced in 1953. In the literature also weighted versions of the Shapley method are considered, i.e. $\Phi^{\alpha}=\sum_{\theta} \alpha(\theta) M^{\theta}$ where $\alpha(\theta) \geq 0$ for any permutation $\theta$ on $N$ and $\sum_{\theta} \alpha(\theta)=1$.

There exist various axiomatic characterizations of the Shapley method. The most well-known characterization is due to Shapley himself (1953) and is as follows:

(a) A cost allocation method $M: C G_{n} \rightarrow \mathbb{R}^{n}$ is efficient, anonymous, additive and possesses the dummy player property if and only if $M=\Phi$.

Here additivity means that $M\left(c^{1}+c^{2}\right)=M\left(c^{1}\right)+M\left(c^{2}\right)$ for all $c^{1}, c^{2} \in C G_{n}$. Recently, Driessen (1985a) proved that another axiomatic characterization of the Shapley method can be obtained by replacing two classical axioms (anonymity and dummy player property) by the two following axioms:

(b1) $M: C G_{n} \rightarrow \mathbb{R}^{n}$ is said to possess the weak dummy player property if $M_{i}(c)$ $=c(\{i\})$ for all $c \in C G_{n}$ and all $i \in N$ for which $c(S \cup\{i\})-c(S)+c(N-S)$ $-c(N-(S \cup\{i\}))=2 c(\{i\})$ whenever $S \subset N-\{i\}$.

(b2) $M: C G_{n} \rightarrow \mathbb{R}^{n}$ is said to possess the equal individuality property if $M_{i}(c)$ $=M_{j}(c)$ for all $c \in C G_{n}$ and all $i, j \in N, i \neq j$ such that $c(S)=c(N-S)$ for all $S \subset N$ with $i \in S$ and $j \notin S$.

Young (1985) showed that also the additivity axiom can be replaced by a monotonicity axiom. Although the Shapley method is monotonic in the aggregate, another form of monotonicity was required by Young:

(c) A cost allocation method $M: C G_{n} \rightarrow \mathbb{R}^{n}$ is efficient, anonymous and strongly monotonic if and only if $M=\Phi$.

Here strongly monotonicity means that $M_{i}\left(c^{2}\right) \geq M_{i}\left(c^{1}\right)$ for all $c^{1}, c^{2} \in C G_{n}$ and all $i \in N$ such that $c^{2}(S \cup\{i\})-c^{2}(S) \geq c^{1}(S \cup\{i\})-c^{1}(S)$ for all $S \subset N$.

Axiomatic characterizations of the Shapley method are also treated in Aumann and Shapley (1974) and Dubey (1975), while axiomatic characterizations of the weighted versions of the Shapley method are treated in Weber (1978). Loehman and Whinston (1974) derive from a set of axioms a charge scheme for public investments. This charge 
scheme coincides with the Shapley cost allocation method on cost functions. Other papers which are closely related to the Shapley method are Hamlen et al. (1980), Jensen (1977) and Loehman and Whinston (1971). The Shapley cost allocation method is applied to many practical situations, for which we refer to $\$ 4.2$ of this paper or to Lucas (1981), Shubik (1962).

\section{(2) Methods Minimizing Maximum Unhappiness}

Pioneering in this field was Schmeidler (1969) with his paper in which he introduced the game theoretical concept nucleolus. Later many authors modified the ideas of Schmeidler and introduced related concepts.

For any cost function $c \in C G_{n}$, its imputation set $I(c)$ consists of all efficient allocations, which are individually rational. Thus

$$
I(c)=\left\{y \in \mathbb{R}^{n} ; \sum_{i \in N} y_{i}=c(N) \text { and } y_{i} \leq c(\{i\}) \text { for all } i \in N\right\} .
$$

Given a cost function $c \in C G_{n}$ such that $I(c) \neq \varnothing$, the key idea is to look first at a certain function $u:\{S ; S \subset N\} \times I(c) \rightarrow \mathbb{R}$, where for a coalition $S$ and an imputation $y \in I(c)$ the number $u(S, y)$ represents the unhappiness of the coalition $S$ with respect to the allocation $y$, and then to minimize the maximum unhappiness. Hence, if for $x, y \in I(c)$

$$
\max \{u(S, x) ; S \subset N\}<\max \{u(S, y) ; S \subset N\}
$$

then $x$ is seen as a better cost allocation than $y$. If in $(*)$ the equality holds (instead of the inequality), then a more subtle look at the unhappiness of coalitions with respect to $x$ and $y$ follows. In general, for any $u:\{S ; S \subset N\} \times I(c) \rightarrow \mathbb{R}$ and any $y \in I(c)$, let $\theta(u, y)$ be the vector in $\mathbb{R}^{2^{n}}$ whose coordinates are the numbers $u(S, y), S \subset N$, arranged in nonincreasing order. Given $c$ and $u$, we then say that $x \in I(c)$ is a better cost allocation than $y \in I(c)$ if $\theta(u, x)$ is lexicographically smaller than $\theta(u, y)$, i.e. $\theta(u, x) \leq_{L} \theta(u, y)$. The $u$-nucleolus of the cost function $c$ is now defined to be the set

$$
N_{u}(c):=\left\{x \in I(c) ; \theta(u, x) \leq_{L} \theta(u, y) \text { for all } y \in I(c)\right\} .
$$

The nucleolus of the cost function $c$ (à la Schmeidler) is obtained whenever $u(S, x)$ $:=\sum_{i \in S} x_{i}-c(S)$ for $S \neq \varnothing$ and $u(\varnothing, x):=0$. Grotte (1970) introduced the normalized nucleolus by considering the unhappiness function $u(S, x):=|S|^{-1}\left(\sum_{i \in S} x_{i}\right.$ $-c(S))$ for $S \neq \varnothing$ and $u(\varnothing, x):=0$. For other nucleoli taking into account the size $|S|$ of a coalition $S$, we refer to Wallmeier (1983). Some other versions of the nucleolus, known as disruption nucleoli, are studied by Gately (1974), Littlechild and Vaidya (1976) and Charnes, Rousseau and Seiford (1978). In Gately (1974) and Littlechild and Vaidya (1976) the unhappiness function for cost games with a nonempty strict core is given by

$$
u(S, x):=\left[\sum_{i \in N-S} x_{i}-c(N-S)\right]\left[\sum_{i \in S} x_{i}-c(S)\right]^{-1} \quad \text { for } S \neq \varnothing, N .
$$

In Charnes et al. (1978), the ratio of these two quantities is replaced by the normalized difference, thus for $S \neq \varnothing, N$

$$
u(S, x):=|N-S|^{-1}\left[c(N-S)-\sum_{i \in N-S} x_{i}\right]-|S|^{-1}\left[c(S)-\sum_{i \in S} x_{i}\right]
$$

Schmeidler (1969) proved that the nucleolus of a cost game consists of a single point and hence it gives rise to a cost allocation method on $C G_{n}$, which turns out to be efficient, individually rational, stable, anonymous and to possess the dummy player property. However, as Megiddo (1974) showed, the nucleolus cost allocation method is not monotonic in the aggregate. An axiomatic characterization of the nucleolus has 
been found by Sobolev (1975). For practical applications of the nucleolus method, we refer to $\S 4.2$ or to Lucas (1981), Suzuki and Nakayama (1976).

\section{(3) Methods Based on Separable and Nonseparable Costs}

In these methods one can distinguish two steps. Given any cost function $c \in C G_{n}$, in the first step to each participant $i$ is allocated his marginal or separable cost, i.e. the cost $m_{i}^{c}:=c(N)-c(N-\{i\})$. Thus the separable cost of player $i$ is the raise in costs if player $i$ joins the coalition $N-\{i\}$. Let $m^{c}=\left(m_{1}^{c}, m_{2}^{c}, \ldots, m_{n}^{c}\right)$. For many cost functions the total of separable costs will be less than the joint cost of the grand coalition, i.e. $\sum_{i \in N} m_{i}^{c}<c(N)$. In the second step the remaining cost

$$
g^{c}(N):=c(N)-\sum_{i \in N} m_{i}^{c}(>0)
$$

is allocated in some way to the participants. The remaining cost $g^{c}(N)$ is called the nonseparable cost. Let $w_{1}, w_{2}, \ldots, w_{n}$ be nonnegative numbers with $\sum_{i=1}^{n} w_{i}>0$, where the weights $w_{i}, i=1,2, \ldots, n$, may be constant but they may also depend on the cost function. Then the separable cost allocation method with weight vector $w=\left(w_{1}, w_{2}, \ldots, w_{n}\right)$ assigns to a cost function $c \in C G_{n}$ the cost allocation

$$
S(c, w)=m^{c}+g^{c}(N)\left(\sum_{i=1}^{n} w_{i}\right)^{-1} w .
$$

Separable cost allocation methods were considered by engineers and economists of the Tennessee Valley Authority (TVA) in the 1930's in relation to apportioning costs of dam systems in the Tennessee River. For more information on this TVA-project, other water resource projects and their corresponding cost allocation problems, we refer to Heaney (1979), Ransmeier (1942), Straffin and Heaney (1981).

Two of the separable cost allocation methods considered by the TVA are the equal charge method (EC-method) and the alternative cost avoided method (ACA-method) where the weight vectors are respectively $(1,1, \ldots, 1)$ and $\left(c(\{1\})-m_{1}^{c}, c(\{2\})\right.$ $\left.-m_{2}^{c}, \ldots, c(\{n\})-m_{n}^{c}\right)$. Hence, the EC-method is given by

$$
\mathrm{EC}(c)=m^{c}+n^{-1} g^{c}(N)(1,1, \ldots, 1)
$$

and the ACA-method is given by

$$
\operatorname{ACA}(c)=m^{c}+g^{c}(N)\left[\sum_{i=1}^{n}\left(c(\{i\})-m_{i}^{c}\right)\right]^{-1}\left(c(\{1\})-m_{1}^{c}, \ldots, c(\{n\})-m_{n}^{c}\right) .
$$

The method commonly used among water resource engineers is the separable cost remaining benefits method (SCRB-method), which is a modification of the ACAmethod. The weight $w=\left(w_{1}, w_{2}, \ldots, w_{n}\right)$ in the SCRB-method depends on the cost function $c$ as follows:

$$
w_{i}=\min \left\{c(\{i\}), b_{i}\right\}-m_{i}^{c} \quad \text { for all } i \in N,
$$

where $b_{i}$ is the benefit to player $i$ if only his purposes are served. Note that for those situations, where $c(\{i\}) \leq b_{i}$ for all $i \in N$, the SCRB-method coincides with the ACAmethod.

The three separable methods mentioned above are efficient, anonymous but not stable since these methods do not take into account the coalitions, for which the number of players is more than 1 but less than $n-1$. In the next section we shall introduce a new separable cost allocation method, which does take into account all coalitions. For studies on the cost allocation problem in water resources development, we refer to Loehman et al. (1979), Loughlin (1977), Young et al. (1982). Legros (1982) 
studies the equivalence of the nucleolus method and the SCRB-method for a certain class of cost games.

\section{The Cost Gap Allocation Method and Its Properties}

In 1981 Tijs introduced the $\tau$-value, a new game theoretical solution concept. In this section we describe the cost allocation method which corresponds to the $\tau$-value. We shall call this method the cost gap method because the allocation of the nonseparable cost by this separable method is determined with the aid of a cost gap function. It will turn out that the EC-, ACA- and SCRB-method, mentioned in the previous section, can be seen as "rough" forms of this cost gap method since they coincide for certain classes of cost functions where the coalitions of size unequal to $1, n-1$ and $n$ are not essential.

Let $\langle N, c\rangle$ be a cost game and $m^{c}=\left(m_{1}^{c}, m_{2}^{c}, \ldots, m_{n}^{c}\right) \in \mathbb{R}^{n}$ the marginal vector for $\langle N, c\rangle$, whose $i$ th coordinate is the separable cost of player $i$, i.e. $m_{i}^{c}=c(N)$ $-c(N-\{i\})$ for all $i \in N$. For each coalition $S$ we define the cost gap of $S$ in the game $\langle N, c\rangle$ by

$$
g^{c}(S):=c(S)-\sum_{i \in S} m_{i}^{c} \quad \text { if } S \neq \varnothing \quad \text { and } \quad g^{c}(\varnothing):=0 .
$$

The map $g^{c}: 2^{N} \rightarrow \mathbb{R}$ is called the cost gap function of the cost game $\langle N, c\rangle$. Note that $g^{c}(N)$ is equal to the nonseparable cost in the cost game. In general we shall assume that the cost gap function is nonnegative.

Let $\langle N, c\rangle$ be a cost game with a nonnegative cost gap function, $i \in N$ and $S \subset N$ such that $i \in S$. As in all separable methods, the separable cost $m_{i}^{c}$ is seen as a lower bound for the cost contribution of player $i$ to the joint cost $c(N)$. Now player $i$ can argue that it is unreasonable that he contributes more than $m_{i}^{c}+g^{c}(S)$ to the joint cost. Namely, if the cost allocation to player $i$ exceeds the amount $m_{i}^{c}+g^{c}(S)$, then he can threaten to try to form the coalition $S$ and to allocate the corresponding cost $c(S)$ in such a way that each other player $j \in S, j \neq i$, has only to pay the lower amount $m_{j}^{c}$, while player $i$ himself contributes the remaining cost $c(S)-\sum_{j \in S-\{i\}} m_{j}^{c}$, which equals $m_{i}^{c}+g^{c}(S)$, so player $i$ would be better off than before.

The above argument holds for any $S$ with $i \in S$. Hence, the number $\min _{S ; i \in S}\left(m_{i}^{c}\right.$ $+g^{c}(S)$ ) or equivalently $m_{i}^{c}+\min _{S ; i \in S} g^{c}(S)$ can be seen as an upper bound for the cost contribution of player $i$ to the joint $\operatorname{cost} c(N)$.

Due to the above reasoning, we define for any cost function $c \in C G_{n}$ a corresponding weight vector $w^{c}=\left(w_{1}^{c}, w_{2}^{c}, \ldots, w_{n}^{c}\right) \in \mathbb{R}^{n}$ by

$$
w_{i}^{c}:=\min _{S ; i \in S} g^{c}(S) \quad \text { for all } i \in N .
$$

Then the number $w_{i}^{c}$ can be seen as the maximal contribution of player $i$ to the nonseparable cost $g^{c}(N)$. We shall now also assume that the total of these maximal contributions covers the nonseparable cost $g^{c}(N)$, i.e. $\sum_{i \in N} w_{i}^{c} \geq g^{c}(N)$.

The cost gap allocation method is now defined as the separable method, where the nonseparable cost is allocated to the players proportional to the abovementioned weight vector. Formally, to any cost function $c \in C G_{n}$ such that

$$
g^{c}(S) \geq 0 \quad \text { for all } S \subset N \quad \text { and } \quad \sum_{i \in N} w_{i}^{c} \geq g^{c}(N)
$$

the cost gap allocation method assigns the cost allocation

$$
\begin{aligned}
C G A(c) & :=m^{c} & & \text { if } g^{c}(N)=0, \\
& =m^{c}+g^{c}(N)\left(\sum_{i \in N} w_{i}^{c}\right)^{-1} w^{c} & & \text { if } g^{c}(N)>0 .
\end{aligned}
$$


The cost allocation $C G A(c)$ can also be obtained as the point where the hyperplane $\left\{y \in \mathbb{R}^{n} ; \sum_{i \in N} y_{i}=c(N)\right\}$ of efficient cost allocations intersects the straight line segment with end points $m^{c}$ and $m^{c}+w^{c}$, as indicated by Figure 1 .

In particular, for cost functions $c \in C G_{n}$ with a nonempty core, the vector $m^{c}$ turns out to be a lower bound and the vector $m^{c}+w^{c}$ an upper bound for the core of $c$, which imply that the cost function $c$ satisfies the conditions in (3.1). Furthermore, it follows from this that the class

$$
Q^{n}:=\left\{c \in C G_{n} ; g^{c}(S) \geq 0 \text { for all } S \subset N \text { and } \sum_{i \in N} w_{i}^{c} \geq g^{c}(N)\right\}
$$

is a full-dimensional cone in the linear space $C G_{n}$. For a proof of these remarks we refer to Tijs (1981), where also a list of properties for the $\tau$-value can be found. The corresponding list for the cost gap method is as follows.

THEOREM 3.1. The cost gap allocation method CGA $: Q^{n} \rightarrow \mathbb{R}^{n}$ is efficient, individually rational and possesses the dummy player property, the anonymity property, the strategic equivalence property and the continuity property.

The cost gap method on $Q^{2}$ or $Q^{3}$ is also stable, but it is not stable on $Q^{n}$ whenever $n \geqslant 4$. A complete list of properties which characterize the cost gap method will appear in Tijs (1986). Some other properties of this method are given in the next theorems. The first result states that the cost allocation to the nondummy players by the cost gap method is not affected if the dummy players in a game are removed.

THEOREM 3.2. (The dummy out property for $C G A: Q^{n} \rightarrow \mathbb{R}^{n}$ ). Let $\langle N, c\rangle$ be a cost game and $D$ the set of dummy players, i.e.

$$
D:=\{i \in N ; c(S \cup\{i\})-c(S)=c(\{i\}) \text { for all } S \subset N-\{i\}\} .
$$

Let $\left\langle N-D, c^{\prime}\right\rangle$ be the cost game with $c^{\prime}(S)=c(S)$ for all $S \subset N-D$. If $c \in Q^{n}$, then $c^{\prime} \in Q^{n-|D|}$ and $C G A_{i}\left(c^{\prime}\right)=C G A_{i}(c)$ for all $i \in N-D$.

Proof. For $i \in D$ we have $m_{i}^{c}=c(N)-c(N-\{i\})=c(\{i\})$ and for $i \in N-D$ : $m_{i}^{c^{\prime}}=c^{\prime}(N-D)-c^{\prime}((N-D)-\{i\})=c(N-D)-c((N-D)-\{i\})=c(N)-c(N$ $-\{i\})=m_{i}^{c}$. So, $m_{i}^{c}=c(\{i\})$ if $i \in D$ and $m_{i}^{c}=m_{i}^{c^{\prime}}$ if $i \in N-D$. It follows that for each $S \subset N$,

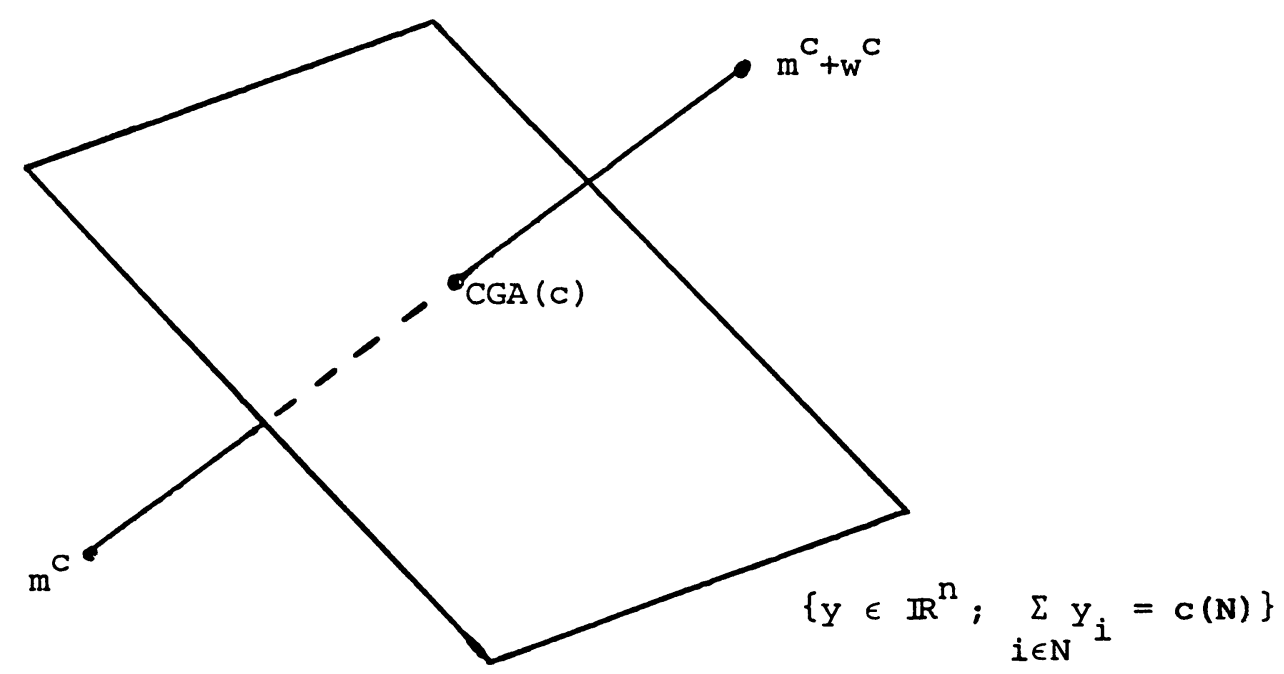

FIGURE 1. 


$$
\begin{aligned}
g^{c}(S) & =c(S)-\sum_{j \in S} m_{j}^{c}=c(S-D)-\sum_{j \in S-D} m_{j}^{c} \\
& =c^{\prime}(S-D)-\sum_{j \in S-D} m_{j}^{c^{\prime}}=g^{c^{\prime}}(S-D) .
\end{aligned}
$$

Thus in particular $g^{c}(N)=g^{c^{\prime}}(N-D)$. But $g^{c}(S)=g^{c^{\prime}}(S-D)$ for all $S \subset N$ implies that $w_{i}^{c^{\prime}}=w_{i}^{c}$ for all $i \in N-D$. Further, for each $S \subset N-D$ we have $g^{c^{\prime}}(S)=g^{c}(S)$ and for all $i \in D$ we have $w_{i}^{c}=0$ since $c \in Q^{n}$ and $i \in D$ imply $0 \leq w_{i}^{c} \leq g^{c}(\{i\})$ $=c(\{i\})-m_{i}^{c}=0$. Hence, $\sum_{j \in N-D} w_{j}^{c^{\prime}}=\sum_{j \in N-D} w_{j}^{c}=\sum_{j \in N} w_{j}^{c}$. Now it follows that $c \in Q^{n}$ implies $c^{\prime} \in Q^{n-|D|}$. Moreover, for the nontrivial case $g^{c}(N)>0$ we have for all $i \in N-D$ :

$$
m_{i}^{c^{\prime}}+g^{c^{\prime}}(N-D)\left(\sum_{j \in N-D} w_{j}^{c^{\prime}}\right)^{-1} w_{i}^{c^{\prime}}=m_{i}^{c}+g^{c}(N)\left(\sum_{j \in N} w_{j}^{c}\right)^{-1} w_{i}^{c} .
$$

Hence, we can conclude that $C G A_{i}\left(c^{\prime}\right)=C G A_{i}(c)$ if $i \in N-D$.

It can be shown that the dummy out property also holds for the cost allocation methods based on the Shapley value and the nucleolus.

The cost gap method is not monotonic in the aggregate, but it possesses another form of monotonicity. The next theorem states that if a cost game $\left\langle N, c_{1}\right\rangle$ is changed to a cost game $\left\langle N, c_{2}\right\rangle$ only by increasing the cost of one coalition $T$ with less than $n-1$ players, then the players outside $T$ are better off in the second game $c_{2}$ than in the original game $c_{1}$ by the cost gap method.

THEOREM 3.3. (Complementary monotonicity for CGA: $Q^{n} \rightarrow \mathbb{R}^{n}$ ). Let $\left\langle N, c_{1}\right\rangle$ be a game and $T \subset N$ with $1 \leq|T|<n-1$. Let $\left\langle N, c_{2}\right\rangle$ be a cost game with $c_{2}(S)$ $=c_{1}(S)$ for all $S \neq T$ and $c_{2}(T) \geq c_{1}(T)$. If $c_{1} \in Q^{n}$, then $c_{2} \in Q^{n}$ and $C G A_{i}\left(c_{2}\right)$ $\leq C G A_{i}\left(c_{1}\right)$ for all $i \in N-T$.

ProOF. Since $|T|<n-1$, we have $m^{c_{2}}=m^{c_{1}}$. This implies that $g^{c_{2}}(S)=g^{c_{1}}(S)$ for all $S \neq T$ and $g^{c_{2}}(T) \geq g^{c_{1}}(T)$. It follows that $w_{i}^{c_{2}}=w_{i}^{c_{1}}$ for $i \in N-T$ and $w_{i}^{c_{2}}$ $\geq w_{i}^{c_{1}}$ for $i \in T$. It is now easy to see that $c_{1} \in Q^{n}$ implies $c_{2} \in Q^{n}$. Further, in case $g^{c_{1}}(N)>0$ we have for all $i \in N-T$ :

$$
m_{i}^{c_{2}}+g^{c_{2}}(N)\left(\sum_{j \in N} w_{j}^{c_{2}}\right)^{-1} w_{i}^{c_{2}}=m_{i}^{c_{1}}+g^{c_{1}}(N)\left(\sum_{j \in N} w_{j}^{c_{2}}\right)^{-1} w_{i}^{c_{1}} \leq m_{i}^{c_{1}}+g^{c_{1}}(N)\left(\sum_{j \in N} w_{j}^{c_{1}}\right)^{-1} w_{i}^{c_{1}}
$$

It follows now that for all $i \in N-T: C G A_{i}\left(c_{2}\right) \leq C G A_{i}\left(c_{1}\right)$.

It is easy to show that the Shapley method on $C G_{n}$ possesses also the complementary monotonicity property, but the nucleolus method does not possess this property (see Driessen 1985b).

Now we consider two subclasses of cost games for which the cost gap method is easy to determine and coincides with other cost allocation methods.

First of all we consider the subclass, consisting of the cost games $\langle N, c\rangle$ satisfying

$$
\min _{S ; i \in S} g^{c}(S)=g^{c}(\{i\}) \geq 0 \quad \text { for all } i \in N .
$$

Games of this type are called semiconvex games and are introduced by Driessen and Tijs (1985). The class of semiconvex $n$-person cost games is a full-dimensional cone in $C G_{n}$, but it is also a subclass of $Q^{n}$. Note that for a semiconvex game $\langle N, c\rangle$ we have for all $i \in N, w_{i}^{c}=g^{c}(\{i\})=c(\{i\})-m_{i}^{c}$ and hence we can state the next result.

THEOREM 3.4. If $\langle N, c\rangle$ is a semiconvex cost game, then $C G A(c)=A C A(c)$.

Most of the cost games which arose in the TVA-project were convex games, which are characterized by the property (see Shapley 1971) 


$$
\begin{array}{ll}
c(S \cup\{i\})-c(S) \geq c(T \cup\{i\})-c(T) & \text { for all } S, T \subset N \text { and } \\
& \text { all } i \in N \text { with } S \subset T \subset N-\{i\} .
\end{array}
$$

This property implies that $c(S \cup\{i\})-c(S) \geq m_{i}^{c}$, or equivalently $g^{c}(S \cup\{i\}) \geq g^{c}(S)$ whenever $i \in N$ and $S \subset N-\{i\}$. From this it follows that convex games are semiconvex.

The second subclass which we want to consider, consists of the cost games $\langle N, c\rangle$ satisfying $\min _{\varnothing \neq S \subset N} g^{c}(S)=g^{c}(N) \geq 0$. These games are called quasi-concave games and are studied in Driessen and Tijs (1983). The class of quasi-concave $n$-person cost games is a full-dimensional cone in $C G_{n}$ and it is also included in $Q^{n}$. These games possess many nice properties, e.g. the cost allocation based on the cost gap method or nucleolus lies in the centre of the core of the game. For a quasi-concave game $\langle N, c\rangle$, we have $w_{i}^{c}=g^{c}(N)$ for all $i \in N$ and hence, the next result is obvious.

THEOREM 3.5. If $\langle N, c\rangle$ is a quasi-concave game, then $C G A(c)=E C(c)$.

We observe that, although the cost gap method on $Q^{n}$ is not stable, this method restricted to the class of quasi-concave $n$-person cost games is stable in such a way that its cost allocation coincides with the barycentre of the core. The same result holds for the Shapley cost allocation method restricted to the class of convex $n$-person cost games (see Shapley 1971). The cost gap method restricted to the class of (semi-)convex $n$-person cost games is stable if $n=2,3$ or 4 , but fails to be stable whenever $n \geq 5$ (see Driessen and Tijs 1985). In the next theorem a weak form of stability for the cost gap method restricted to the class of convex cost games is treated. First we prove a lemma dealing with dummy players. A player $i$ is called a dummy player in a cost game $\langle N, c\rangle$ if $c(S \cup\{i\})-c(S)=c(\{i\})$ for all $S \subset N-\{i\}$.

LEMMA 3.6. Let $\langle N, c\rangle$ be a cost game such that the imputation set I(c) contains at least two points. Then there exist at least two players, who are no dummy players.

PROOF. Let $D$ be the set of the dummy players in the game $c$. Suppose that $|N-D|$ $<2$. Then $|D| \geq n-1$, which implies together with the dummy player definition that $c(S)=\sum_{i \in S} c(\{i\})$ for all $S \neq \varnothing$. But then $I(c)=\{(c(\{1\}), c(\{2\}), \ldots, c(\{n\}))\}$ which contradicts the assumption in the lemma. It follows that there exist at least two nondummy players.

Convex cost games possess a nonempty core (Shapley 1971). Thus, in case the cost gap allocation of a convex cost game falls outside its core, we can conclude by Lemma 3.6 that there are at least two nondummy players in the convex cost game. Now we formulate the announced theorem.

THEOREM 3.7. (Weak stability). Let $\langle N, c\rangle$ be a convex cost game and suppose that $C G A(c) \notin C O R E(c)$. Let $D$ be the set of the dummy players in $c$ and $T \subset N$ such that $\sum_{j \in T} C G A_{j}(c)>c(T)$. Then for each pair $i, j \in T-D, i \neq j$, there exists $x \in C O R E(c)$ such that $x_{i}>C G A_{i}(c)$ and $x_{j}<C G A_{j}(c)$.

Proof. Let $i, j \in T-D, i \neq j$ and $\theta: N \rightarrow N$ a permutation such that $\theta(1)=i$ and $\theta(n)=j$. Let $x \in \mathbb{R}^{n}$ be defined by

$$
x_{\theta(k)}:=c(\{\theta(1), \theta(2), \ldots, \theta(k)\})-c(\{\theta(1), \theta(2), \ldots, \theta(k-1)\}) \quad \text { for all } k \in N .
$$

Since $c$ is convex, $x \in \operatorname{CORE}(c)$ (see Shapley 1971). Note that $x_{i}=c(\{i\})-c(\varnothing)$ $=c(\{i\})$ and $x_{j}=c(N)-c(N-\{j\})=m_{j}^{c}$. It suffices now to show that

$$
m_{k}^{c}<C G A_{k}(c)<c(\{k\}) \quad \text { for all } k \in N-D \text {. }
$$


The convexity of $c$ implies for all $S \subset N$ and all $k \in N-S$,

$$
c(\{k\}) \geq c(S \cup\{k\})-c(S) \geq c(N)-c(N-\{k\})=m_{k}^{c} .
$$

From this follows that $k \in D$ iff $c(\{k\})=m_{k}^{c}$. Hence, $c(\{k\})-m_{k}^{c}>0$ whenever $k \in N-D$.

We know that $C G A(c) \in I(c)$ but $C G A(c) \notin C O R E(c)$, so $I(c)$ contains at least two points, which implies that $c(N)<\sum_{k \in N} c(\{k\})$. By the convexity of $c$ we have that $c \in Q^{n}$, so $g^{c}(S) \geq 0$ for all $S \subset N$. Then $g^{c}(N)=0$ would imply that $m^{c} \in \operatorname{CORE}(c)$ and $m^{c}=C G A(c)$. Because it is given that $C G A(c) \notin C O R E(c)$ we can conclude that $g^{c}(N)>0$. Since convex games are semiconvex, the cost allocation $C G A(c)$ is given by

$$
C G A_{k}(c)=m_{k}^{c}+\alpha\left(c(\{k\})-m_{k}^{c}\right) \quad \text { for all } k \in N
$$

where $\alpha:=g^{c}(N)\left[\sum_{l=1}^{n}\left(c(\{l\})-m_{l}^{c}\right)\right]^{-1}$. Then $\alpha>0$ since $g^{c}(N)>0$, but also $\alpha<1$ since $c(N)<\sum_{k \in N} c(\{k\})$. Let $k \in N-D$. Then $c(\{k\})-m_{k}^{c}>0$ and since $0<\alpha$ $<1$ we obtain that $m_{k}^{c}<C G A_{k}(c)<c(\{k\})$.

An interpretation of Theorem 3.7 can be given. Let $\langle N, c\rangle$ be a convex cost game and suppose that coalition $T$ is not content with the cost gap allocation $C G A(c)$ since $\sum_{j \in T} C G A_{j}(c)>c(T)$ and, hence, some nondummy players in $T$ may threaten to form a subcoalition. Then the arbitrator who proposes the cost gap allocation as a suitable cost allocation can make a counter-threat by looking for a stable allocation $x \in \operatorname{CORE}(c)$, where one of the nondummy players involved will be worse off and another will be better off than his cost gap allocation, without saying beforehand who are the favoured and harmed players.

We conclude this section with the remark that an extension of the cost gap method on $Q^{n}$ to the set $C G_{n}$ of all cost functions is given by the authors (1984).

\section{Examples}

In this last section we compare some cost allocation methods for the two-person cost allocation problems and further, we consider two practical cost allocation problems.

\subsection{Two-Person Cost Allocation Problems}

It is easy to verify that for any two-person cost game $\langle N, c\rangle$ the cost gap allocation coincides with the Shapley and nucleolus allocation and that it is given by

$$
C G A(c)=\frac{1}{2}(c(\{1\})-c(\{2\})+c(\{1,2\}),-c(\{1\})+c(\{2\})+c(\{1,2\})) .
$$

As noted before, it belongs always to the core of $c$.

\subsection{User Fees in the Airport Game}

Cost allocation problems can arise from situations in which some service is provided to a variety of different customers who differ in the amount or type of service they need. One can think of toll roads, computers, telephones and airports. Let us concentrate on the aircraft landing fee problem as discussed in Owen (1982).

Consider an airport with one runway and divide the planes which are to land there into $m$ types. Let $N_{j}$ be the set of landings by planes of type $j$ over a fixed time-span. Then $N:=\bigcup_{j=1}^{m} N_{j}$ is the set of all landings. Let $n_{j}$ denote the number of landings by planes of type $j$ and $n=\sum_{j=1}^{m} n_{j}$.

The cost of building a runway depends upon the largest plane for which the runway is designed. Let $t_{j}$ be the cost to make the airport suitable for landings by planes of 
type $j$. We suppose that $0=t_{0}<t_{1}<t_{2}<\cdots<t_{m}$. The cost function $c$ of the corresponding cost game $\langle N, c\rangle$ is then determined by $c(\varnothing)=0$ and for all $\varnothing \neq S \subset N$

$$
c(S):=\max \left\{t_{j} ; 1 \leq j \leq m, S \cap N_{j} \neq \varnothing\right\} .
$$

The airport economists Baker (1965) and Thompson (1971) proposed the following simple cost allocation rule: each user contributes equally to the cost $t_{1}$; each user, except those of type 1 , contributes equally to $t_{2}-t_{1}$; etc. This means that a user of type $j$ has to pay the amount $\sum_{k=1}^{j}\left(t_{k}-t_{k-1}\right)\left(\sum_{r=k}^{m} n_{r}\right)^{-1}$. Littlechild and Owen (1973) showed that the above-mentioned cost allocation coincides with the cost allocation based on the Shapley value. Dubey (1982) gave a nice axiomatic characterization of the Shapley cost allocation method restricted to such airport cost functions. For the nucleolus of an airport game we refer to Littlechild (1974), Littlechild and Owen (1976), Owen (1982). Here we shall calculate the cost allocation to the users according to the cost gap method. We consider only the practical case where $n_{m} \geq 2$. Then the separable cost of any user is equal to zero, and hence $g^{c}(S)=c(S)$ for all $S \subset N$. In particular, $g^{c}(N)=c(N)=t_{m}>0$. Further, $w_{i}^{c}=\min _{S ; i \in S} g^{c}(S)=t_{j}$ for $i \in N_{j}$. Now it follows that $c \in Q^{n}$ and that the landing fee, based on the cost gap method, for a plane $i$ of type $j$ is equal to

$$
C G A_{i}(c)=t_{m}\left(\sum_{k=1}^{m} n_{k} t_{k}\right)^{-1} t_{j} \quad \text { for } i \in N_{j}
$$

Thus the cost gap allocation is proportional to the cost numbers $t_{j}, 1 \leq j \leq m$, corresponding to the various types.

\subsection{Minimum Cost Spanning Tree Games}

Let $G$ be a network with node set $N \cup\{0\}$. Each node $i \in N$ corresponds to a consumer and the node 0 to a central supplier of some commodity. Let for an arc $(i, j)$, the number $d(i, j)$ denote the cost of connecting $i$ to $j$. For each nonempty coalition $S \subset N$, let $c(S)$ be the minimum cost required to connect all the consumers to the central supplier in the network with node set $S \cup\{0\}$. If we interpret $d(i, j)$ as the length of arc $(i, j)$, then $c(S)$ is the length of a shortest spanning tree of the network with node set $S \cup\{0\}$. There are a lot of papers dealing with cost allocation problems arising from such network situations (see Bird 1976, Claus and Granot 1976, Claus and Kleitman 1973, Granot and Huberman 1981, 1984). It is proved in Granot and Huberman (1981) that the core of a corresponding cost game is nonempty. This

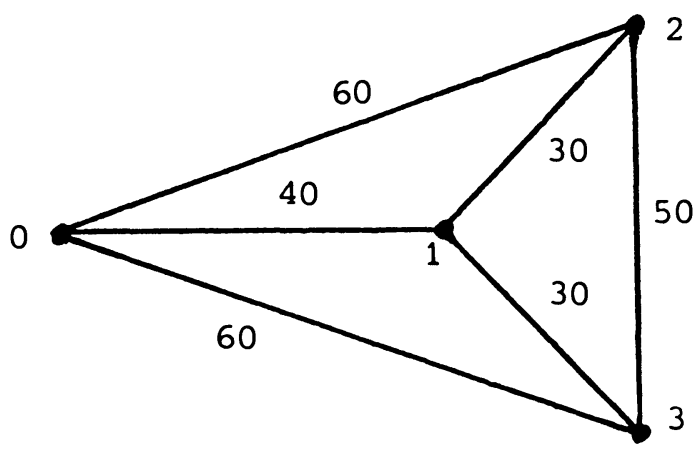

FIGURE 2. 


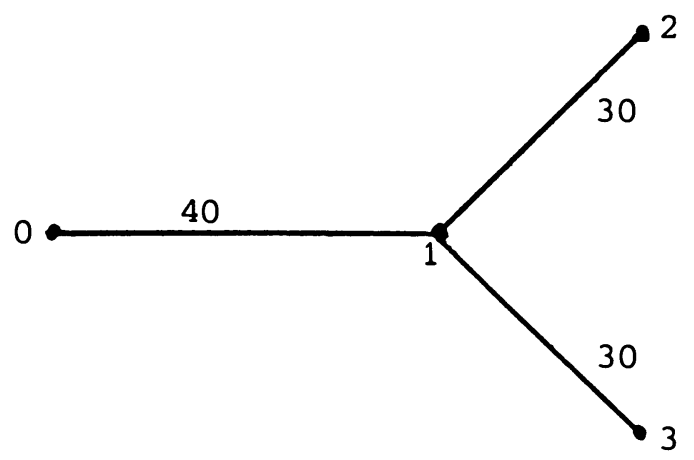

FIGURE 3.

contrasts with the results for Steiner tree games studied in Megiddo (1978), where an example of a 5-person Steiner tree game with an empty core is given.

Let us look at the specific spanning tree game with three consumers, corresponding to the network of Figure 2. The corresponding minimum cost spanning tree game $\langle N, c\rangle$ is determined by $N=\{1,2,3\}$ and $c(\varnothing)=0, c(\{1\})=40, c(\{2\})=c(\{3\})$ $=60, c(\{1,2\})=c(\{1,3\})=70, c(\{2,3\})=110$ and $c(\{1,2,3\})=100$. Here the minimum cost spanning tree for $N$ is given in Figure 3. For this specific spanning tree game, the three cost allocations based on the Shapley value, the nucleolus and the $\tau$-value, are as follows:

the Shapley cost allocation $\Phi(c)=\left(13 \frac{1}{3}, 43 \frac{1}{3}, 43 \frac{1}{3}\right)$,

the nucleolus cost allocation $N(c)=(10,45,45)$,

the cost gap allocation $C G A(c)=\left(12 \frac{8}{11}, 43 \frac{7}{11}, 43 \frac{7}{11}\right)$.

All three of them belong to the core of the game. ${ }^{1}$

${ }^{1}$ The authors would like to thank Patrick Legros for putting our attention to the TVA-project.

\section{References}

Arrow, K. J., Social Choice and Individual Values, John Wiley, New York, 1963.

AumanN, R. J. AND L. S. Shapley, Values of Non-Atomic Games, Princeton University Press, Princeton, N.J., 1974.

BAKER, M. J. AND ASSOCIATES, "Runway Cost Impact Study," Report presented to the Association of Local Transport Airlines, Jackson, Miss., 1965.

BIRD, C. G., “On Cost Allocation for a Spanning Tree: A Game Theoretic Approach,” Networks, 6 (1976), 335-350.

Charnes, A., J. Rousseau AND L. Seiford, "Complements, Mollifiers and the Propensity to Disrupt," Internat. J. Game Theory, 7 (1978), 37-50.

Claus, A. AND D. GRANOT, "Game Theory Application to Cost Allocation for a Spanning Tree," Working paper No. 402, Faculty of Commerce and Business Administration, University of British Columbia, 1976.

AND D. J. KLEITMAN, “Cost Allocation for a Spanning Tree,” Networks, 3 (1973), 289-304.

Driessen, T. S. H., "A New Axiomatic Characterization of the Shapley Value," Methods Oper. Res., 50 (1985a), 505-517.

," Contributions to the Theory of Cooperative Games: The $\tau$-Value and $k$-Convex Games," Ph.D. Thesis, Catholic University, Nÿmegen, The Netherlands, $1985 \mathrm{~b}$.

AND S. H. TIJs, "The $\tau$-Value, the Core and Semiconvex Games," Internat. J. Game Theory, 14 (1985), 229-247.

AND - "The $\tau$-Value, the Nucleolus and the Core for a Subclass of Games," Methods Oper. Res., 46 (1983), 395-406.

AND - "Extensions and Modifications of the $\tau$-Value for Cooperative Games," In: Selected Topics in Operations Research and Mathematical Economics, G. Hammer and D. Pallaschke (Eds.), Springer Verlag, Berlin and New York, 1984, 252-261. 
DubEY, P., "On the Uniqueness of the Shapley Value," Internat. J. Game Theory, 4 (1975), 131-139.

, "The Shapley Value as Aircraft Landing Fees-Revisited," Management Sci., 28 (1982), 869-874.

Gately, D., "Sharing the Gains from Regional Cooperation: A Game Theoretic Application to Planning Investment in Electric Power," Internat. Econom. Rev., 15 (1974), 195-208.

Granot, D., "A Note on the Room-Mates Problem and a Related Revenue Allocation Problem," Management Sci., 30 (1984), 633-643.

AND G. Huberman, "Minimum Cost Spanning Tree Games,” Math. Programming, 21 (1981), $1-18$.

- AND - "On the Core and Nucleolus of Minimum Cost Spanning Tree Games," Math. Programming, 29 (1984), 323-347.

GrotTe, J. H., "Computation of and Observation on the Nucleolus, and the Central Games," M.Sc. thesis, Cornell University, Ithaca, N.Y., 1970.

Hamlen, S. S., W. A. Hamlen, JR. AND J. T. Tschirhart, "The Use of Core Theory in Evaluating Joint Cost Allocation Schemes," Accounting Rev., 52 (1977), 616-627.

, - AND - "The Use of the Generalized Shapley Allocation in Joint Cost Allocation," Accounting Rev., 55 (1980), 269-287.

Heaney, J. P., "Efficiency/Equity Analysis of Environmental Problems: A Game Theoretic Perspective," In: Applied Game Theory, S. J. Brams, A. Schotter and G. Schwodiauer (Eds.), Physica-Verlag, Vienna, 1979, 352-369.

JENSEN, D. L., “A Class of Mutually Satisfactory Allocations,” Accounting Rev., 52 (1977), 842-856.

Legros, P., "The Nucleolus and the Cost Allocation Problem," Report, Evanston, Ill., 1982.

Littlechild, S. C., "A Simple Expression for the Nucleolus in a Special Case," Internat. J. Game Theory, 3 (1974), 21-29.

, "Common Costs, Fixed Charges, Clubs and Games," Rev. Economic Stud., 42 (1975), 117-124.

AND G. OwEN, "A Simple Expression for the Shapley Value in a Special Case," Management Sci., 20 (1973), 370-372. 5 (1976), 91-95.

- AND G. F. ThOMPSON, “Aircraft Landing Fees: A Game Theory Approach,” Bell. J. Economics, 8 (1977), 186-204.

- AND K. G. VAIDYA, "The Propensity to Disrupt and the Disruption Nucleolus of a Characteristic Function Game,” Internat. J. Game Theory, 5 (1976), 151-161.

Loehman, E. And A. Whinston, "A New Theory of Pricing and Decision-Making for Public Investment," Bell J. Econom. and Management Sci., 2 (1971), 606-625.

AND -, "An Axiomatic Approach to Cost Allocation for Public Investment," Public Finance Quart., 1 (1974), 236-251.

, E. Orlando, J. Tschirhart and A. Whinston, "Cost Allocation for a Regional Waste-Water Treatment System,” Water Resources Res., 15 (1979), 193-202.

Loughlin, J. C., "The Efficiency and Equity of Cost Allocation Methods for Multipurpose Water Projects," Water Resources Res., 13 (1977), 8-14.

LuCAS, W. F., "Applications of Cooperative Games to Equitable Allocation," In: Proc. Sympos. Appl. Math., 24, W. F. Lucas (Ed.), Amer. Math. Soc., Providence, R.I., 1981, 19-36.

MEGIDDO, N., "On the Nonmonotonicity of the Bargaining Set, the Kernel and the Nucleolus of a Game," SIAM J. Appl. Math., 27 (1974), 355-358.

, "Cost Allocation for Steiner Trees," Networks, 8 (1978), 1-6.

Owen, G., Game Theory, second ed., Academic Press, New York, 1982.

Ransmeier, J. S., "The Tennessee Valley Authority: A Case Study in the Economics of Multiple Purpose Stream Planning," The Vanderbilt University Press, Nashville, Tenn., 1942.

SCHMEIDleR, D., "The Nucleolus of a Characteristic Function Game," SIAM J. Appl. Math., 17 (1969), 1163-1170.

Shapley, L. S., “A Value for n-Person Games,” Ann. of Math. Studies, 28 (1953), 307-317.

- "Cores of Convex Games," Internat. J. Game Theory, 1 (1971), 11-26.

SHubiK, M., "Incentives, Decentralized Control, the Assignment of Joint Cost and Internal Pricing," Management Sci., 8 (1962), 325-343.

Sobolev, A. I., "The Characterization of Optimality Principles in Cooperative Games by Functional Equations," In: Mathematical Methods in Social Sciences 6, N. Vorobjev (Ed.), Academy of Sciences of the Lithuanian SSR, Vilnius, 1975, 94-151.

Straffin, P. D. AND J. P. HeANEY, "Game Theory and the Tennessee Valley Authority," Internat. J. Game Theory, 10 (1981), 35-43.

SUZUKI, M. AND M. NAKAYAMA, "The Cost Assignment of the Cooperative Water Resource Development: A Game Theoretical Approach,” Management Sci., 22 (1976), 1081-1086. 
ThOMPSON, G. F., Airport costs and pricing. Unpublished Ph.D. dissertation, University of Birmingham, 1971.

Tus, S. H., "Bounds for the Core and the $\tau$-Value," In: Game Theory and Mathematical Economics, O. Moeschlin and D. Pallaschke (Eds.), North-Holland Publ. Cie., Amsterdam, 1981, 123-132. , "An Axiomatization of the $\tau$-Value," Math. Social Sci. (to appear in 1986).

AND F. A. S. LiPPERTS, "The Hypercube and the Core Cover of $n$-Person Cooperative Games," Cahiers Centre Études Rech. Opér., 24 (1982), 27-37.

WALlmeiER, E., "Der f-Nukleolus und ein dynamisches Verhandlungsmodell als Lösungskonzepte für kooperative $n$-Personenspiele," Dissertations nachdruck, Skripten zur Mathematischen Statistik, 5, Universität Münster, 1983.

Weber, R. J., "Probabilistic Values for Games," Cowles Foundation Discussion Paper No. 471R, Yale University, New Haven, Conn., 1978.

Young, H. P., "Monotonic Solutions of Cooperative Games," Internat. J. Game Theory, 14 (1985), 65-72. , N. OKADA AND T. HAShimoto, "Cost Allocation in Water Resources Development," Water Resources Res., 18 (1982), 463-475. 